\title{
Pricing Diagnostic Information
}

\author{
Ashish Arora \\ Carnegie Mellon University, Hamburg Hall 1108, 5000 Forbes Avenue, Pittsburgh, Pennsylvania 15213, \\ ashish@andrew.cmu.edu \\ Andrea Fosfuri \\ Universidad Carlos III de Madrid, Madrid 126-28903, Getafe, Madrid, Spain, and \\ Center for Economic Policy Research (CEPR), London, United Kingdom, fosfuri@emp.uc3m.es
}

\begin{abstract}
$D$ iagnostic information allows an agent to predict the state of nature about the success of an investment project better than the prior. We analyze the optimal pricing scheme for selling diagnostic information to buyers with different, privately known, ex ante success probability. Investment costs and returns of successful projects are assumed to be the same for all buyers. The value of diagnostic information is the difference in expected payoffs with and without it, and we show that the willingness to pay for diagnostic information is nonmonotonic in the ex ante success probability. When the information seller can offer only one quality level, and negative payments are not allowed, we find that the optimal menu of (linear) contracts is remarkably simple. A pure royalty is offered to buyers with low ex ante success probability, and a pure fixed fee is offered to buyers with high ex ante success probability.
\end{abstract}

Key words: pricing; diagnostic information; marketing research

History: Accepted by Jagmohan S. Raju, marketing; received October 8, 2003. This paper was with the authors 4 months for 2 revisions.

\section{Introduction}

It is good management practice to acquire information before committing the firm to an important decision or a major investment. External sources of such information include marketing and strategic consultants, legal advisors, information technology (IT) consultants, financial research firms, auditors, and the growing number of information services companies that offer electronic access to proprietary databases. Individuals also solicit information from a variety of sources before taking important decisions. For instance, buyers of used cars sometimes invest in a diagnostic check by a trusted mechanic or acquire online information about the specific model and the history of the vehicle from a service such as Carfax. Other examples include property evaluation by real estate experts, medical advice, and legal counsel.

This paper analyzes how to price diagnostic information, which is defined as information that is: (1) valuable for making decisions, and (2) unbundled from physical objects, services, and execution. In some cases, diagnostic information comes bundled with execution. For instance, doctors, IT consultants, and management consultants are typically paid both for diagnosing the problem and also for solving it. Similarly, many accounting and audit products both scan for errors and also rectify them. We do not deal with these cases here. In a seminal paper, Arrow (1962) modeled (diagnostic) information as a signal that changes the estimates of the probabilities of the different states of nature, allowing an informed agent to make better decisions. Arrow's work provides a crucial insight: Diagnostic information has economic value only insofar as the decision maker changes his actions as a consequence of the revised probabilities in at least one state of the world. ${ }^{1}$

From a practical viewpoint, how information services are priced is important. Selling information that is later used in decision making is a growing business (Jensen 1991). Professional consulting services are exemplars of the growing importance of the diagnostic information market, which has increased at more than $10 \%$ a year during the 1980 s. Marketing research consultants have the lion's share of the industry. The top 50 U.S. market research firms reported worldwide revenues of $\$ 5.96$ billion in 1998. Market research analysts held about 135,000 jobs in the United States in 2002, with employment expected to grow by more than 36\% through 2012 (http://www. bls.gov). Market research firms provide clients with

\footnotetext{
${ }^{1}$ Information, in addition to affecting the estimates of the probabilities of the different states of nature, can also help identify new alternatives that were unknown a priori. Although this is an important component of the value of information, scholars have tended to disregard it because of the difficulty of putting a value on a priori nonexisting states of nature. In this paper, we will follow in this tradition and focus on information as modifying estimated probabilities of known states of the world.
} 
information to decide on the promotion, distribution, design, and pricing of products or services. Marketing information may also be used in decisions such as adding new product lines or opening new branches. Other examples of diagnostic information include opinion services by portfolio analysts, legal counseling without further representation, and patent validity or patent infringement opinions by lawyers. ${ }^{2}$ For simplicity, in what follows we shall simply use information to mean diagnostic information.

To focus on how pricing is affected by the value of information, we develop a simple model where an agent has to decide whether or not to invest in a project with an uncertain outcome and can purchase information that will update his beliefs about the outcome. The value of information is the difference in expected payoffs with and without it. Information buyers are assumed to be heterogeneous in the ex ante success probability. We show that the value of information is nonmonotonic in the ex ante success probability. Indeed, information is more valuable to buyers who have the highest a priori uncertainty between investing in the project or not.

We then analyze the optimal pricing for a monopolist offering a single information quality. Our model's simplifying features, namely that all buyers invest the same amount (if they invest) and receive the same payoff (if the project is successful), imply that we can restrict ourselves to linear contracts. The optimal menu of contracts is remarkably simple. If negative payments are not allowed, a pure royalty is offered to buyers with low ex ante success probability and a pure fixed fee is offered to buyers with high ex ante success probability. The fact that the optimal menu of contracts collapses to only two contracts is due to both the linearity of contracts and the piecewise linearity of the value of information. To grasp the intuition behind our finding, note that whereas a pure fixed fee implies that the payment for the information is independent of the ex ante expected value of the project, a royalty means that the payment is contingent on the success of the project and therefore increasing in its ex ante expected value. A contingent payment is therefore especially ill suited to capture value from buyers with high ex ante expected value projects, for whom information is not that important.

The simplicity of the optimal menu of contracts has important practical implications. It explains why we do not observe complex pricing schemes for selling

\footnotetext{
${ }^{2}$ Firms often consult patent lawyers to assess whether their product infringes on another's patent, or if another patent is valid, or both. The lawyers that provide such opinions are almost always not employed by the firm, and moreover, typically will not represent the firm if the latter is sued for patent infringement (Lemley and Tangri 2003).
}

information. Managers do not have to search for elaborate and complex formulae; instead, they need focus only on finding the optimal values for the royalty and fixed fee. Of course, these implications apply to those information markets whose reality approximates the special features of our model. For simplicity, we ignore whether the information is sold as a service or embodied in a software or hardware diagnostic tool, which may affect whether the buyer can "resell" the information to others. The way in which information is sold may also affect the cost structure of the seller. We follow the literature in assuming that the marginal cost of serving an additional buyer is zero. In the next section, we position our paper in the context of the related literature. Section 3 derives the value of diagnostic information. Section 4 analyzes optimal pricing in the basic model with homogenous information, whereas $\$ 5$ explores the robustness of the basic result to extensions and generalizations. Section 6 concludes the paper. The appendix, available online (http://mansci.pubs.informs.org/ecompanion. $h t m l)$, contains the proofs.

\section{Related Literature}

This paper is related to several streams of the managerial and economic literature. Within the informationmarketing literature, the papers closest to ours are Iyer and Soberman (2000) and Chang and Jevons Lee (1994), which analyze the optimal selling and pricing policies for a marketing consultant who sells information that increases the value of the downstream products. Because buyers' characteristics are common knowledge (an assumption we relax in our paper), the consultant can extract the full value of information from each buyer. However, because buyers interact in the product market, their willingness to pay depends upon which other buyers also have the information. Thus, the optimal pricing scheme consists of selecting the subset of buyers to whom to sell information. By contrast, we ignore interactions in the downstream market and focus on information that is nonrival and can be used by several buyers without loss of value. Formally, we assume that the values that buyers derive from information are independent. This implies that our analysis does not directly apply to cases where exclusive access to the information is an advantage. In turn, our paper derives the value of information from the underlying characteristics of the investment project and generates greater insights about the contractual mechanisms upon which a market for information is based.

To the best of our knowledge, the only paper that has explicitly modeled diagnostic information is Sarvary (2002). He looks at the market for second 
opinions and analyzes competition between information sellers. He assumes that the sellers are differentiated in that they offer information with different levels of precision and shows that there is an equilibrium in which the low-quality seller sells first and then (depending on the signal received) the buyer can buy the high-quality information with some probability. Differently from our paper, he assumes common knowledge about the buyer's willingness to pay for the information and does not deal with pricing issues. Competition among information sellers is also analyzed by Sarvary and Parker (1997) in a game-theoretic model that consists of two firms selling information to a population of buyers who are heterogeneous in their willingness to pay for information quality. They analyze how the nature of competition is affected by the reliability of information and the correlation between different sources of information. As we do, they assume that there are no interactions in the downstream market. Unlike us, however, they ignore the possibility of offering a menu of contracts to buyers. In sum, within the informationmarketing literature, our paper is the first to analyze the optimal pricing mechanism for selling diagnostic information to heterogeneous buyers with privately known ex ante values for the information. ${ }^{3}$

Pricing issues have also been addressed by the recent literature on "information products" or "digital products." Sundararajan (2004) analyzes optimal pricing of digital goods under incomplete information when both fixed-fee and royalty pricing are feasible. He shows that offering fixed-fee pricing in addition to a usage-based pricing scheme is always (weakly) profit improving. Jain and Kannan (2002) examine various pricing schemes for information products sold online. They look at three common pricing schemes for access to proprietary databases: connecttime-based pricing, search-based pricing, and subscription pricing. They show that search-based pricing is typically preferred to connect-time-based pricing, and that subscription pricing might be used in combination with search-based pricing when the value of information varies across users. Although similar in spirit, our paper differs in three important aspects. First, we focus on the pricing of information in the Arrow sense: information that is unbundled from physical objects or services, whose value derives solely from improved decision making. Second, we derive the value of information, whereas they assign it. Third, we identify and characterize the optimal

\footnotetext{
${ }^{3}$ Raju and Roy (2000) use a game-theoretic model to study how industry characteristics moderate the effect of market information on firm profits.
}

menu of contracts for the information seller, whereas they simply compare different pricing schemes. ${ }^{4}$

\section{A Model for Diagnostic Information}

Consider a consulting company (the consultant) selling reports to clients (the buyers) who use these reports to make decisions about investment projects. ${ }^{5}$ Each buyer can only undertake one project, by investing a fixed amount $I$. Projects can either be good $(G)$ or bad $(B)$. A $G$ project generates a (gross) return of $V>0$, whereas a $B$ project generates a return of 0 . There is an ex ante probability $q$ that a given project is of type $G$, and $1-q$ that it is of type $B$.

An important ingredient of our model is that buyers are heterogeneous. We assume that buyers, indexed by $q$, are distributed according to the distribution function $F(q)$. We also assume that the support of $F(q)$ is compact, $f(q)$ is the density function, and that $q \in[0,1]$. The consultant does not observe $q$, which could reflect unobserved differences in the buyer's ability or unobserved differences in the market conditions facing the buyer, but does observe its distribution $F$. One interpretation of $q$ is as follows. Suppose that firms use heuristics to screen projects to cull less-promising ones. Projects are indexed by the probability of success, $p$, which has a distribution $h(p)$. If a firm is able to screen out and reject projects with $p<p^{*}$, then $q=\int_{p^{*}}^{1} p h(p) d p$ is the conditional expectation of the success probability associated with the set of projects that survive the screening process. Heterogeneity in the ability of the firms to screen out projects is represented by $p^{*} .6$

For instance, in the case of marketing research information, $V$ could be the net benefit of launching a new product, $I$ the cost of adding a new line to the existing product offer, and $q$ the ex ante probability that the new product will be successfully accepted by the final consumers. Without new information, a buyer

\footnotetext{
${ }^{4}$ There are other related papers as well, albeit with a very different focus. For example, Bashyam (2000) analyzes duopolistic competition in the supply of information products, but focuses on the selection of the distribution channel-online versus CD-ROM-rather than the pricing mechanisms. Bakos and Brynjolfsson (1999) examine whether firms should bundle different information goods and charge one bundle price.

${ }^{5}$ The assumption of a monopolist information seller implies that there is a high fixed cost for acquiring and processing proprietary information and a negligible marginal cost for information dissemination. For instance, IMS International, a subsidiary set up by Dun \& Bradstreet in 1954, is the leading supplier of proprietary information about the sales of pharmaceutical and other health care products (Chang and Jevons Lee 1994).

${ }^{6}$ Alternatively, one can assume that the source of heterogeneity across buyers is $V$, the gross return of a successful project, which yields qualitatively similar results, but is analytically less tractable.
} 
Table 1

Modeling Diagnostic Information

\begin{tabular}{lcc}
\hline & Good project $(G)$ & Bad project $(B)$ \\
\hline Positive signal $(Y)$ & $\operatorname{Pr}(Y \mid G)$ & $\operatorname{Pr}(Y \mid B)$ \\
Negative signal $(N)$ & $\operatorname{Pr}(N \mid G)$ & $\operatorname{Pr}(N \mid B)$ \\
\hline
\end{tabular}

invests if $q V>I$. Information produces a signal about the type of the project (see Table 1). After observing the signal, the buyer updates his beliefs and decides whether or not to invest.

Let $\operatorname{Pr}(Y \mid G)=m>0.5$ and $\operatorname{Pr}(N \mid B)=n>0.5$. Hence, $\operatorname{Pr}(N \mid G)=(1-m)$ and $\operatorname{Pr}(Y \mid B)=(1-n)$. An $N$ signal conditional on a $G$ project is also referred to as a "false negative," whereas a $Y$ signal conditional on a $B$ project is typically called a "false positive." Note that the "quality" of the information is given by $m$ and $n$. Larger values of $m$ and $n$ imply a smaller probability of false negative and false positive, i.e., the signal is more reliable. Using Baye's Law, the probability that the project is of type $G$ given a $Y$ signal and given $q$ is

$$
\operatorname{Pr}(G \mid Y, q)=\frac{m q}{m q+(1-q)(1-n)} \quad \text { and }
$$

the probability that the project is of type $G$ given an $N$ signal and given $q$ is

$$
\operatorname{Pr}(G \mid N, q)=\frac{(1-m) q}{(1-m) q+(1-q) n} \cdot ?
$$

\subsection{The Value of Information: Nonmonotonicity}

The value of information for a given buyer is the difference between his expected profits with information and his expected profits without it. Differences in $q$ translate into differences in the private benefit of information. However, as we shall see, higher $q$ buyers do not necessarily benefit the most from having the information. Because information has value insofar as it changes decisions, we need to consider two separate cases depending on whether or not the buyer would have invested without information.

Case 1. $q V \geq I$, the buyer invests even without information. In this case, the buyer values information because it reduces the probability of a bad investment

\footnotetext{
${ }^{7}$ The quality or accuracy of the information ( $m$ and $n$ ) is assumed to be exogenous; the content of the information, i.e., the probability of getting a $Y$ signal, does depend upon $q$. If one treats $q$ itself as a random variable, then it follows that the signal and $q$ are correlated. Specifically, $\operatorname{Pr}(Y \mid q)=m q+(1-n) q$, and if $q$ is assumed to be uniform between $[0,1]$, then $\operatorname{Pr}(Y)=m / 2+(1-n) / 2$, so that $\operatorname{Cov}(Y, q)=(m+n-1) / 12>0$. If $q$ is treated as nonstochastic, then the distribution of the signal depends on $q$. This dependence does not raise any problems because the contracting takes place before the consultant observes any signal. Thus, at the time of contracting, the consultant has no additional information about $q$.
}

Figure 1 Nonmonotonicity in the Value of Information

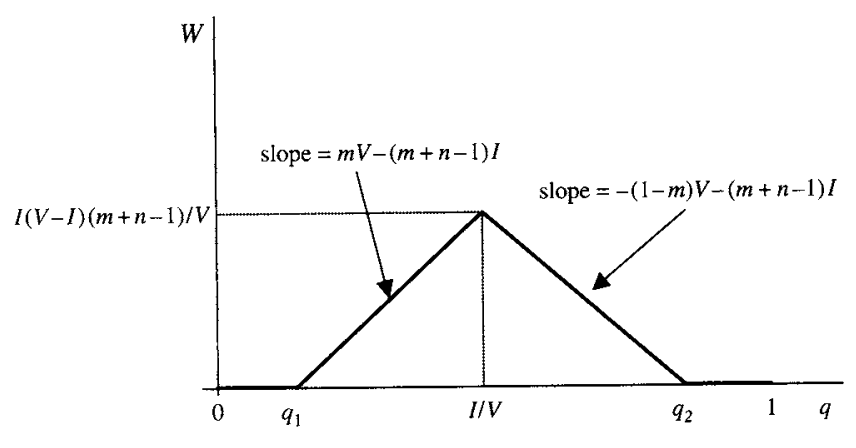

decision. Given that $m>0.5$, the buyer invests only if there is a $Y$ signal, so that the expected profit is $\operatorname{Pr}(G \mid Y, q) \operatorname{Pr}(Y \mid q)(V-I)+\operatorname{Pr}(B \mid Y, q) \operatorname{Pr}(Y \mid q)(-I)$, whereas the expected profit without information is $(q V-I)$. The difference between the two is the value of information, i.e.,

$$
\begin{aligned}
W(q) & =q m(V-I)+(1-q)(1-n)(-I)-(q V-I) \\
& =n I-q[(1-m) V+(m+n-1) I] .
\end{aligned}
$$

Note that $W(q)$ is equal to zero at

$$
q=\frac{n I}{V(1-m)+(m+n-1) I}=q_{2}
$$

For any $q>q_{2}, \operatorname{Pr}(B \mid N, q) V-I \geq 0$, so that even an $N$ signal does not change the buyer's action (invest), and therefore information is valueless to such buyers.

Case 2. $q V<I$, the buyer does not invest without information. The value of information is given by $W(q)=$ $q m(V-I)+(1-q)(1-n)(-I)$. (In this case, the expected profit of the buyer without information is zero.) One can rewrite this expression as $W(q)=$ $-(1-n) I+q[m V-(m+n-1) I]$. Note that $W(q)$ is equal to zero at

$$
q=\frac{(1-n) I}{m V-(m+n-1)}=q_{1} .
$$

For any $q<q_{1}, \operatorname{Pr}(G \mid Y, q) V-I \leq 0$, so that even a $Y$ signal does not change the buyer's action (not invest), and therefore information is valueless to such buyers.

Figure 1 shows graphically the value of information. The intuition behind this graph is the following. For $q \geq I / V, W(q)$ represents the saving in investment costs, $n I(1-q)$, minus the loss from a false negative (relative to the baseline of "invest"), $(1-m)(V-I) q$. For this range of parameter values, the buyer values information because it could prevent (with some probability) a bad investment. As $q$ rises, the cost of a false negative rises and the saving in investment cost decreases. For Case 2, $W(q)$ represents the gain from investing (the baseline is "not invest"), $m q(V-I)$, 
minus the loss from a false positive, $(1-n)(1-q) I$. Note that the gain is increasing in $q$, and the cost of a false positive is decreasing in $q$. In this range, the buyer values information because it could suggest (with some probability) that investing is a good decision.

Proposition 1. The value of information is nonmonotonic in $q$. It increases in $q$ for $q \leq I / V$ and decreases in $q$ thereafter.

This is an intuitive result. ${ }^{8}$ Information is more valuable to buyers who have the highest a priori uncertainty between investing or not in the project. Buyers who have either a highly negative or a highly positive ex ante expected value of the project are less likely to change their actions as a consequence of the revised probabilities, and hence will pay less for the information. Inasmuch as options are a substitute for information, this result is also consistent with the conventional finding that the value of an option increases with the variance of the underlying asset's value (Dixit and Pindyck 1994).

REMARK 1 . The value of information is strictly increasing in both $m$ and $n$, decreasing in $V$ and increasing in $I$ if $q>I / V$, and increasing in $V$ and decreasing in $I$ if $q<I / V$. Additionally, the value of information is strictly increasing in $\gamma$, where $\gamma$ is a scale factor that multiplies both $V$ and $I$.

More precise information is more valuable. Also, when the underlying decision is more important (i.e., higher $V$ and $I$ ), then information is more valuable.

REMARK 2. For $q>I / V, d W(q) / d m>d W(q) / d n$, and for $q<I / V, d W(q) / d m<d W(q) / d n$.

In other words, all buyers on the right of $I / V$ (see Figure 1) are relatively more concerned with the probability of a false negative, whereas all buyers on the left of $I / V$ are relatively more concerned with the probability of a false positive. Indeed, for $q>I / V$, the baseline is to invest. These buyers are going to change their actions only if they observe an $N$ signal. Hence, they would like the $N$ signal be as precise as possible, i.e., the probability of a false negative, $\operatorname{Pr}(N \mid G)=$ $1-m$, to be small.

\section{Optimal Pricing of Diagnostic Information}

We assume that the consultant offers only one information quality and that such a quality is observable to the buyers. We discuss relaxing the first assumption in $\$ 5$, and briefly discuss the latter here. In some

\footnotetext{
${ }^{8}$ The standard assumption in the literature on price discrimination (see Tirole 1988, Chapter 2) is that the buyers' willingness to pay is a monotonic function of their "type." As we show here, this assumption is implausible for diagnostic information.
}

cases, observing the quality of information may not be difficult. For instance, information might be embodied in a specific piece of equipment or research technology whose characteristics are well understood. In other cases, although the quality of information is not directly observable, legal or reputation reasons might produce a similar effect. However, when the production of information involves human judgment based on expertise or experience (e.g., management advice), the quality of information is more difficult to assess. This case raises adverse selection problems. In addition, the consultant can also have incentives to distort the information if he is also involved in fixing the diagnosed problem or if (as is the case here) he receives a contingent payment. In this paper, we abstract from these important problems in information economics (Arrow 1962, Emons 1997, Dye and Sridhar 2003).

Menu of Contracts. We assume that contracts are offered to buyers before the signal is observed and can include payments contingent on whether the project succeeds. Given that $q$ is not observed by the consultant, any contract can either have a fixed payment or one that is contingent on success, or both. Because every buyer has the same $V$ and $I$, together these observations imply that any contract can be represented as a linear contract that combines a fixed fee and a payment contingent on success, i.e., royalties. Hence, let a contract be defined by the pair $\{\alpha, \beta\}$, where $\alpha$ represents a fixed fee and $\beta$ represents a royalty. We model the royalty as a share $\beta$ of project's (gross) returns, $V$, paid only if the project succeeds. ${ }^{9}$ A pure fixed-price contract and a pure royalty contract are therefore defined as $\{\alpha, 0\}$ and $\{0, \beta\}$, respectively. A pure fixed price means that the payment for the information does not vary with $q$. A royalty implies that the payment for the information is an increasing function of $q$. Hence, low $q$ buyers tend to prefer a royalty scheme, whereas high $q$ buyers tend to prefer a fixed price. Moreover, a royalty is especially ill suited to capture value from high $q$ buyers, because for these buyers the value of information is decreasing in $q$. We assume throughout the paper

\footnotetext{
${ }^{9}$ If investment decisions were observable, then contracts could also specify payments contingent on investment taking place. Note that investment occurs with probability $m q+(1-n)(1-q)$. Hence, for any given royalty $t$ and a fixed price $\lambda$, each potential buyer $q$ would pay $\lambda+(1-n) t I+q(m+n-1) t I$. However, this can be replicated with an appropriate two-part tariff, $\alpha=\lambda+(1-n) t I$ and $\beta=(m+n-1) t I / V m$. A qualitatively similar alternative is to model outcome-contingent contracts as profit-sharing contracts where the consultant gets a share of the net returns, $V-I$. All contingent payment schemes create incentives for the consultant to distort information, a possibility that we rule out by assumption.
} 
Figure 2 Fixed Price and Royalty

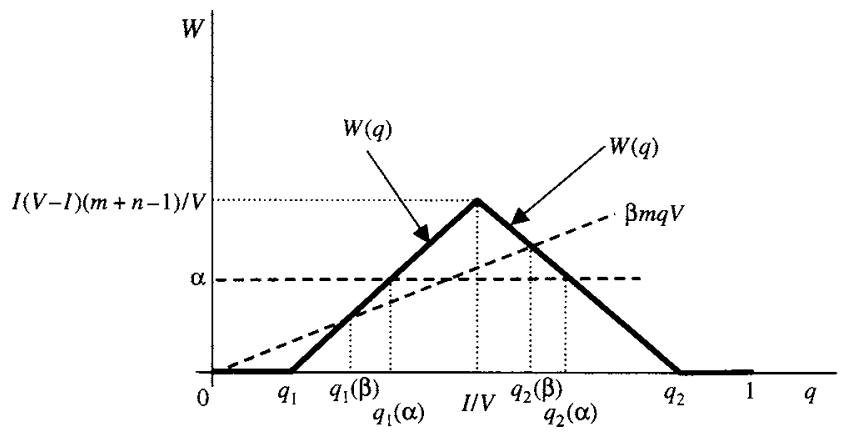

that $\alpha$ and $\beta$ are nonnegative. ${ }^{10}$ Figure 2 shows an arbitrary fixed fee and an arbitrary royalty.

A menu of contracts is a set of such pairs $\{\alpha, \beta\}$, possibly one for each $q$. We first characterize the optimal menu of contracts and then we compute it under a special case. Note that the characterization of the optimal menu of contracts does not rely on any assumption about $F(q)$, the distribution of $q$. First, it is useful to establish the following two properties of the optimal menu of contracts.

LEMMA 1. Let $\{\alpha(q), \beta(q)\}, \alpha(q), \beta(q) \in \Re^{+}$, be the contract designed for buyer $q$.

(a) Then, for any $q^{\prime}>q, \alpha(q) \leq \alpha\left(q^{\prime}\right)$ and $\beta(q) \geq \beta\left(q^{\prime}\right)$.

(b) Concavity. Take $q_{1}, q_{2}, q_{3}$ such that $q_{1}<q_{2}$, $q_{3}=\lambda q_{1}+(1-\lambda) q_{2}$ with $0 \leq \lambda \leq 1$. Then, $\lambda\left[\alpha\left(q_{1}\right)+\right.$ $\left.\beta\left(q_{1}\right) m V q_{1}\right]+(1-\lambda)\left[\alpha\left(q_{2}\right)+\beta\left(q_{2}\right) m V q_{2}\right] \leq \alpha\left(q_{3}\right)+$ $\beta\left(q_{3}\right) m V q_{3}$.

Lemma 1 implies that the amount of payments that the consultant can extract with an optimal menu of contracts must be an increasing and concave function of $q$. Buyers with larger ex ante expected values of the project would be asked to pay a higher price.

Proposition 2 (Characterization of the OptIMal Menu of Contracts). If $\alpha, \beta \in \mathfrak{R}^{+}$, then the optimal menu of contracts consists of the following two contracts: a pure royalty $\left\{0 ; \beta_{1}\right\}$ and a pure fixed price $\left\{\alpha_{2} ; 0\right\} .\left\{0 ; \beta_{1}\right\}$ is chosen by all $q \in\left[q_{1}\left(\beta_{1}\right), \tilde{q}\left(\beta_{1}, \alpha_{2}\right)\right]$ and $\left\{\alpha_{2} ; 0\right\}$ is chosen by all $q \in\left(\tilde{q}\left(\beta_{1}, \alpha_{2}\right), q_{2}\left(\alpha_{2}\right)\right]$, where

$$
\begin{aligned}
q_{1}\left(\beta_{1}\right)= & \frac{(1-n) I}{m\left(1-\beta_{1}\right) V-(m+n-1) I}, \\
q_{2}\left(\alpha_{2}\right)= & \frac{n I-\alpha_{2}}{(1-m) V+(m+n-1) I}, \quad \text { and } \\
& \tilde{q}\left(\beta_{1}, \alpha_{2}\right)=\frac{\alpha_{2}}{\beta_{1} m V} .
\end{aligned}
$$

\footnotetext{
${ }^{10}$ Negative prices are typically not observed in practice and raise a number of difficulties. For instance, if the consultant pays a buyer to use the diagnostic information and the latter only pays if they invest (or succeed), this may attract fraud; even those without any investment project would claim to be buyers, thereby hoping to get paid by the consultant.
}

Figure 3 The Optimal Menu of Contracts

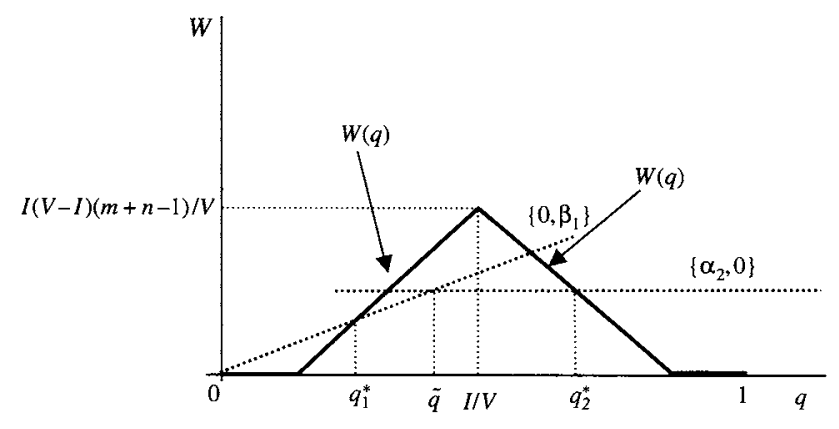

Figure 3 illustrates the optimal menu of contracts. The intuitive argument of why the optimal menu of contracts collapses to only two contracts is as follows. Let $q_{1}^{*}$ and $q_{2}^{*}$ be the highest and the lowest $q$ that buy the information. Given that $\alpha, \beta>0$, each contract defines a straight line with (weakly) positive intercept and slope. Consider the two lines that correspond to the contracts offered, respectively, to $q_{1}^{*}$ and $q_{2}^{*}$. Due to incentive compatibility, these two lines mark the upper bound of what can be extracted from any buyer $q \in\left(q_{1}^{*}, q_{2}^{*}\right)$. The consultant cannot obtain higher profits by offering other (different) contracts to the rest of the buyers. Hence, the optimal menu of contracts has only two contracts. It is now simple to see why these two contracts must be a pure royalty and a pure fixed fee. Suppose that, instead of a fixed fee, the consultant uses a two-part tariff to extract value from $q_{2}^{*}$. This contract would mark an upper bound of what can be extracted from any $q<q_{2}^{*}$, that is, below that defined by a fixed-fee contract. This would reduce the possibilities of value extraction, and therefore cannot be optimal. A similar argument could be put forward for the pure royalty contract. Finally, note that the finding that high $q$ buyers are charged a fixed fee and low $q$ buyers are charged a royalty is rather intuitive too. Indeed, whereas a fixed fee implies that the payment for the information is independent of the ex ante expected value of the project, a royalty means that the payment is contingent on the success of the project and therefore increasing in its ex ante expected value. A contingent payment is therefore especially ill suited to capture value from buyers with high ex ante expected value projects, for whom information is not that important. The result in Proposition 2 depends upon key elements of our model: (a) the value of information is nonmonotonic in $q$; (b) buyers do not choose how much to invest in the project and all successful projects have the same return, so that the value of information is (piecewise) linear in $q$; (c) the consultant can offer only one quality level; and (d) negative payments are disallowed. In $\S 5$, we discuss the robustness of Proposition 2.

This finding has important practical implications. It suggests that when restricted to linear contracts, the 
design of the optimal menu of contracts is rather simple (Figure 3). It suffices to offer to the buyers the choice between a royalty-based contract and a fixedfee contract, and let the buyers self-select. Buyers with low ex ante expected value of the project (who also care more about false positives) would choose the royalty-based contract, whereas buyers with high ex ante expected value of the project (who also care more about false negatives) would choose the fixedfee contract. Hence, the optimization problem of the consultant collapses to the choice of suitable values for the royalty and the fixed fee.

One can write the consultant's profits as follows:

$$
\Pi^{M}=\int_{q_{1}\left(\beta_{1}\right)}^{\tilde{q}\left(\beta_{1}, \alpha_{2}\right)} \beta_{1} m V q f(q) d q+\int_{\tilde{q}\left(\beta_{1}, \alpha_{2}\right)}^{q_{2}\left(\alpha_{2}\right)} \alpha_{2} f(q) d q,
$$

where

$$
\begin{gathered}
q_{1}\left(\beta_{1}\right)=\frac{(1-n) I}{m\left(1-\beta_{1}\right) V-(m+n-1) I}, \\
q_{2}\left(\alpha_{2}\right)=\frac{n I-\alpha_{2}}{(1-m) V+(m+n-1) I}, \text { and } \\
\tilde{q}\left(\beta_{1}, \alpha_{2}\right)=\frac{\alpha_{2}}{\beta_{1} m V} .
\end{gathered}
$$

Solving for the first-order conditions, one can compute the optimal value of $\beta_{1}$ and $\alpha_{2}$, provided one knows the distribution of $q$ and the values of $V$ and $I \cdot{ }^{11}$

Remark 3 (Comparative Statics). Let $q$ be uniformly distributed between 0 and 1 . Then, $\partial \beta_{1} / \partial V>0$, $\partial \alpha_{2} / \partial V>0, \partial \beta_{1} / \partial I<0, \partial \alpha_{2} / \partial I<0, \partial \beta_{1} / \partial \gamma=0$, and $\partial \alpha_{2} / \partial \gamma>0$, where $\gamma$ is a scale factor that multiplies both $V$ and $I$.

The comparative statics with respect to $\gamma$ are easily understood if one thinks of a change in $\gamma$ as a change in the unit of measure of both $V$ and $I$. Whereas the fixed price, also measured in monetary units, should change accordingly, the royalty, being a share, should not be affected.

Remark 4 (Comparative Statics: Quality of INFORMATION). Let $q$ be uniformly distributed between 0 and 1 . Then, $\partial \beta_{1} / \partial m>0, \partial \alpha_{2} / \partial m>0, \partial \beta_{1} / \partial n>0$, and $\partial \alpha_{2} / \partial n>0$. Let $m+n=$ constant. Then, $\partial \beta_{1} / \partial m<$ 0 and $\partial \alpha_{2} / \partial m<0$.

As expected, information of better quality commands both a higher fixed fee and a higher royalty in the optimal menu of contracts. However, if one keeps constant the overall quality of the signal (i.e., the sum of false positives and false negatives is held

\footnotetext{
${ }^{11}$ For instance, let $q$ be uniformly distributed between 0 and $1, V=$ $\$ 4 \mathrm{~m}$ and $I=\$ 1 \mathrm{~m}$. The probability of a false positive and that of a false negative are both $20 \%$. Then, the optimal menu of contracts is formed by a royalty of $35.4 \%$ and a fixed fee of $\$ 247,400$. The expected profits for the consultant will amount to $\$ 80,000$ per buyer.
}

constant), then information with a lower false positive rate is sold through a menu of contracts that involves a larger fixed fee and a larger royalty. The reason for this finding comes from two arguments. First, for any acceptable royalty, the optimal fixed fee is increasing in the level of the royalty because the residual demand is less elastic. Indeed, if the royalty is high, there are few buyers that switch to the royalty if the consultant increases the fixed fee. Second, low $q$ buyers tend to prefer a royalty and are relatively more concerned with the probability of a false positive. So, if the false positive rate decreases, these buyers would obtain higher value from the information, and the consultant would optimally increase the level of the royalty, which in turn would also imply a higher fixed fee.

\section{Robustness and Model Extensions}

We briefly discuss here the assumptions that lead to the major finding of this paper; that is, the optimal menu of contracts to sell diagnostic information is composed of two simple contracts, one contingent royalty for low $q$ buyers, and a fixed fee for high $q$ buyers. ${ }^{12}$

First, note that we have assumed that both the royalty and the fixed fee must take nonnegative values. If one removes this assumption, then it is easy to show that the optimal menu of contracts allows full value extraction, and it is formed by two two-part tariff contracts. Indeed, by precisely fine tuning the royalty and the fixed fee, one can design a contract that coincides with the value of information for all $q<I / V$ and another contract that does the same for all $q>I / V$. As noted earlier, allowing money transfers from the consultant to the buyer is unrealistic and creates perverse incentives for buyers.

Second, contracts are linear in $q$ because $V$ and $I$ are constant across buyers. Linear contracts are standard in the literature and also correspond to the common practice in the industry. Third, the linearity of the value of information in $q$ is critical for our results. The literature has most often analyzed models where the value of information is assumed to be concave in the buyer's type. Maintaining the nonmonotonicity result, let $W(q)$ be continuous, twice-differentiable, and concave between $q_{1}$ and $q_{2}$, with a unique maximum at $q=I / V$ and $W\left(q_{1}\right)=W\left(q_{2}\right)=0$. Under the assumption that both the royalty and the fixed fee cannot take negative values, it is easy to see that the highest and the lowest $q$ that buy the information will be offered a pure royalty and a pure fixed fee (one can actually perform the same type of reasoning provided in the intuition of Proposition 2). Depending

\footnotetext{
${ }^{12}$ For the algebra, see our working paper at http://www.heinz.cmu.
} $\mathrm{edu} /$ wpapers $/$ detail.jsp?id $=4364$. 
on the slope of the value of information, these two contracts might again turn out to be the only two contracts available in the optimal menu. However, there exist parameter configurations under which one can show that for a compact and closed set of $q$ (strictly smaller than $q_{2}^{*}$ ), the consultant offers a continuum of two-part tariff contracts, each of them tangent to the value of information.

Because we do not assume the value of information, but we derive it from the underlying decision framework, it is important to understand how one could obtain a value of information that is concave in $q$ in our model. Note that we have assumed that information does not affect the level of investment, but only whether the investment is actually undertaken or not. The level of investment could be a decision variable for the buyer as well. If one assumes that $I$ is a decision variable and that $V$ is an increasing concave function of $I$, then the amount invested by a buyer depends on $q$ and on the information available to the buyer. Under specific functional forms one can show that the value of information is concave and nonmonotonic in q. However, in this scenario the royalty affects the returns of the investment, and, in turn, how much the buyer will invest. Therefore, the royalty has an additional inefficiency absent in our basic model. Finding the optimal menu of contracts under this scenario proved intractable. We do not think that this would make the optimal menu of contracts necessarily more complex. However, the present model falls short of providing a thorough verification of this conjecture that, we hope, will be addressed in future research.

Finally, we assume a single quality of information. In the literature, typically buyers with higher (marginal) willingness to pay receive higher quality (or quantity) of the good or service. In principle, information could be differentiated vertically (i.e., higher or lower overall precision) as well as horizontally (i.e., different combinations of false positives and false negatives). Differentiated quality heightens the problems of moral hazard on the part of the consultant if the quality is not observed by the buyer. Sarvary (2002) provides a treatment of vertically differentiated quality. Instead, we analyzed the case of horizontally differentiated quality, where the consultant offers two different qualities of information, i.e., signals with different combinations of $m$ and $n$, with $m+n$ held constant. Although the optimal menu of contracts changes, its main feature remains similar to that obtained in Proposition 2. That is, high $q$ buyers will be charged a fixed fee and low $q$ buyers will be charged a pure royalty. For $q$ in-between, either a fixed fee or a pure royalty may be charged, depending on parameter values. Further, high $q$ buyers purchase the signal with the smaller probability of a false negative and low $q$ buyers purchase the signal with the smaller probability of a false positive. Obviously, increasing the number of different qualities of information available leads to an expansion of the optimal menu of contracts, but once again, each element of the optimal menu is either a pure royalty or a pure fixed fee. We conjecture that allowing for more variants will yield similar results: more pricing options, but each option being either a pure fixed fee or a pure royalty.

We have explored the robustness of our result in other ways as well. In an early version of this paper, we analyzed a specification of the model where buyers' heterogeneity came through the returns of a good project rather than the probability of a good project. This specification is less tractable, but nonetheless yields similar results.

We also analyzed an extension where we have competition among two consultants. When the consultants sell identical quality, the standard Bertrand result holds where price competition leads to zero profits. However, when the information is differentiated, i.e., each consultant has a different combination of $m+n$, we find that consultants tend to choose different pricing mechanisms to alleviate competition.

\section{Conclusion}

Diagnostic information that simply changes the estimates of the probabilities of the different states of nature has economic value if the decision maker would change his actions as a consequence of the revised probabilities. However, trading information is not easy, and although Arrow (1962) envisaged the possibility of information becoming a commodity, he also warned about the impediments to markets for information. We do not dispute the existence of such problems. However, as the examples such as marketing research, independent financial research, and legal advice have shown, there are markets, albeit imperfect, for information. In this paper, we have tried to understand how these markets work and, in particular, how information is priced.

We have found that the buyer's willingness to pay for information is nonmonotonic in the buyer's "type," implying that the standard "revelation mechanism" solution cannot be mechanically applied. However, the optimal pricing scheme for a monopolist information owner turned out to be extremely simple. A pure royalty is offered to buyers whose projects have low ex ante expected value and a pure fixed fee is offered to buyers whose projects have high ex ante expected value.

Subject to the caveats discussed earlier, this finding has important practical implications. In particular, when diagnostic information affects mainly whether an investment takes place, but not the size 
of the investment, so that the value of information is approximately linear, our results suggest that consultants do not need complicated pricing schemes to maximize profits. It suffices to offer the buyers a choice between a royalty-based contract and a fixedfee contract, and let the buyers self-select. Buyers with low ex ante expected value of the project would choose the royalty-based contract, whereas buyers with high ex ante expected value of the project would choose the fixed-fee contract. Hence, the optimization problem of the seller collapses to the choice of suitable values for the royalty and the fixed fee.

An online appendix to this paper is available at http://mansci.pubs.informs.org/ecompanion.html.

\section{Acknowledgments}

The authors thank an anonymous associate editor, two reviewers, S. Brusco, J. S. Raju (department editor, marketing), T. Roende, D. Soskice, and participants at the EPRIS first annual meeting (London 2000) and at the EARIE conference (Dublin 2001) for helpful comments and suggestions on an earlier draft. Andrea Fosfuri gratefully acknowledges financial support from the European Commission (HPSECT-2002-00146) and the Spanish Ministry of Science and Technology (SEC2003-03797). This research was conducted when Andrea Fosfuri was visiting the Software Industry Center at the Heinz School of Public Policy and Management, Carnegie Mellon University, whose hospitality is gratefully acknowledged. The usual disclaimer applies.

\section{References}

Arrow, K. J. 1962. Economic welfare and the allocation of resources for inventions. R. Nelson, ed. The Rate and Direction of Inventive
Activity: Economic and Social Factors. Princeton University Press, Princeton, NJ.

Bakos, Y., E. Brynjolfsson. 1999. Bundling information goods: Pricing, profits and efficiency. Management Sci. 45(12) 1613-1630.

Bashyam, T. C. A. 2000. Service design and price competition in business information services. Oper. Res. 48(3) 362-375.

Chang, C. H., C. W. Jevons Lee. 1994. Optimal pricing strategy in marketing research consulting. Internat. Econom. Rev. 35(2) 463-478.

Dixit, A. K., R. S. Pindyck. 1994. Investment Under Uncertainty. Princeton University Press, Princeton, NJ.

Dye, R. A., S. S. Sridhar. 2003. Investment implications of information acquisition and leakage. Management Sci. 49(6) 767-783.

Emons, W. 1997. Credence goods and fraudulent experts. RAND J. Econom. 28(1) 107-119.

Iyer, G., D. Soberman. 2000. Markets for product modification information. Marketing Sci. 19(3) 203-225.

Jain, S., P. K. Kannan. 2002. Pricing of information products on online servers: Issues, models, and analysis. Management Sci. 48(9) 1132-1142.

Jensen, F. O. 1991. Information services. C. A. Congram, M. L. Friedman, eds. The AMA Handbook of Marketing for the Service Industries. AMA-COM, New York.

Lemley, W. A., R. K. Tangri. 2003. Ending patent law's willfulness game. Public Law Research Paper 142, University of California at Berkeley, Berkeley, CA.

Raju, J. S., A. Roy. 2000. Market information and firm performance. Management Sci. 46(8) 1075-1084.

Sarvary, M. 2002. Temporal differentiation and the market for second opinions. J. Marketing Res. 34 129-136.

Sarvary, M., P. M. Parker. 1997. Marketing information: A competitive analysis. Marketing Sci. 16(1) 24-38.

Sundararajan, A. 2004. Non-linear pricing of information goods. Management Sci. 50(12) 1660-1673.

Tirole, J. 1988. The Theory of Industrial Organization. MIT Press, Cambridge, MA. 